\title{
Calidad técnico cientifica del cuidado de enfermería en el Programa de Planificación Familiar
}

\section{Scientific technical quality of nursing care in Family Planning Program}

\author{
Alexandra García-R ${ }^{1}$; Martha-I Camargo-R ${ }^{1}$; María-T Fajardo- $\mathrm{P}^{1}$
}

Forma de citar: García A, Camargo M, Fajardo MT. Calidad técnico científica del cuidado de enfermería en el Programa de Planificación Familiar. Rev Univ Ind Santander Salud. 2018; 50(1): 47-57. doi: http://dx.doi.org/10.18273/revsal.v50n1-2018005 (c) (i)

\section{Resumen}

Introducción: Identificar la aplicación de los requerimientos técnico científicos que los profesionales de enfermería tienen en cuenta en el desarrollo del programa de planificación familiar, es importante para orientar la calidad del cuidado y la reducción de riesgos que amenazan la salud de usuarios, acorde con el ideal de atención en salud sexual y reproductiva. Objetivo: Identificar el cumplimiento del profesional de enfermería de los aspectos técnicocientíficos del programa de planificación familiar, en una institución de salud. Materiales y métodos: Estudio de corte transversal. Población: usuarios del programa de planificación familiar. Muestra: 345 usuarias, 11 enfermeros. Se aplicó un instrumento que evalúa la calidad del cuidado brindado en relación con: la humanización del cuidado, satisfacción con la atención, cumplimiento de aspectos técnico - científicos y disponibilidad de recursos. Procesamiento y análisis de datos: epidata 3, Stata v12. Resultados: El 97,9\% de los profesionales diligencia adecuadamente los formatos de historia clínica y los hallazgos identificados en la consulta. El $98 \%$ de las usuarias no recibió asesoría en derechos sexuales y reproductivos. En el 98,5\% de los casos de inserción de implante subdérmico, se aplicó el consentimiento informado a usuarias. El 84\% de las usuarias no recibió información sobre: el uso del preservativo como método de doble protección, la importancia del autoexamen de mama el $90 \%$, ni de la citología el $60,5 \%$. Conclusiones: Es notoria la falta de información a usuarias sobre cuidados sexuales y reproductivos y los derechos relacionados con estos cuidados. Fue positivo el cumplimiento de aspectos éticos en la atención.

Palabras clave: Calidad de la atención en salud; Planificación familiar; Enfermería.

\begin{abstract}
Introduction: Identifying the technical requirements that nursing professionals must abide when developing the family planning program, is relevant for the quality of care and the reduction of patients' risks, as well as users's health, according to the ideal of sexual and reproductive healthcare. Objective: To identify the nursing professionals' compliance of the technical and scientific aspects of the family planning program in a health institution. Materials
\end{abstract}

1. Universidad Industrial de Santander. Bucaramanga, Colombia.

Correspondencia: Alexandra García. Dirección: Escuela de Enfermería. Facultad de Salud. Carrera 32 No. 29-31. Universidad Industrial de Santander. Correo electrónico: alexagar@uis.edu.co. Teléfono: +576344000 Ext.3016. 
and methods: Cross sectional study. Population: Users of the family planning program. Sample: 345 users, 11 nurses. An instrument was implemented for evaluating the quality of care provided in relation to humanization of healthcare, satisfaction with healthcare, compliance with technical and scientific aspects, and availability of resources. Data processing and analysis was performed with Epidata 3, Stata v12. Results: $97.9 \%$ of professionals adequately handled the clinical history formats and the findings identified in the consultation. $98 \%$ of the users did not receive counselling on sexual and reproductive rights. In $98.5 \%$ of cases of subdermal implant insertion, informed consent was obtained. $84 \%$ of the users did not receive information about condom use as a double protection method, $90 \%$ about the importance of breast self-examination, and $60 \%$ of cytology. Conclusions: It is notorious the lack of information to users on sexual and reproductive care and the rights related to this care. The fulfillment of ethical aspects in healthcare was positive.

Keywords: Quality of health care; Family planning; Nursing.

\section{Introducción}

La conferencia de población y desarrollo llevada a cabo en El Cairo en 1994, estableció el compromiso de los diferentes países del mundo con el derecho de los hombres y las mujeres a estar informados y a tener acceso a métodos de su elección de planificación de la familia que sean seguros, eficaces, costeables y aceptables, así como a otros métodos de su elección para la regulación de la fecundidad, que no violen la $l^{1}{ }^{1}$, aspectos que implican al interior de los sistemas de salud políticas orientadas a la adherencia de los usuarios a los programas y servicios que se ofrecen por parte de los profesionales del sector.

Es así como el estado colombiano desde hace más de una década ha incorporado el sistema de garantía de la calidad, que define la calidad de la atención en salud, como la provisión de servicios a los usuarios individuales y colectivos de manera accesible y equitativa, a través de un nivel profesional óptimo, teniendo en cuenta el balance entre beneficios, riesgos y costos, con el propósito de lograr la adhesión y satisfacción de dichos usuarios $^{2}$.

Teniendo en cuenta que el profesional de enfermería tiene la responsabilidad en la atención en planificación familiar a hombres y mujeres ${ }^{3}$, se torna esencial hacer evidente la calidad con la cual está llevando a cabo este tipo de programa en el ámbito científico técnico.

Estas condiciones de capacidad tecnológica y científica, son los requisitos básicos de estructura y de proceso que deben cumplir los proveedores de Servicios de Salud durante la atención que prestan y que se consideran suficientes y necesarios para reducir los principales riesgos que amenazan la vida o la salud de los usuarios en el marco de la prestación del servicio de salud, como pueden ser embarazos no deseados, abortos, períodos intergenésicos cortos, multiparidad, embarazo adolescente entre otros.

Si bien es cierto, en Colombia se cuenta con una normatividad enfocada a garantizar la calidad de la atención en salud $\mathrm{d}^{4-5}$, por parte de las instituciones prestadoras de servicios y el personal de salud, las cuales son de obligatorio cumplimiento; aún existen necesidades no cubiertas relacionadas con la aplicación de dicha normatividad, que deben ser identificadas con el fin de establecer planes de mejora orientados al cumplimiento del deber ser de la planificación familiar, entendida como el conjunto de actividades, procedimientos e intervenciones dirigidas a hombres y mujeres en edad fértil, dentro de los cuales se encuentra la información, educación, consejería en anticoncepción, incluyendo la entrega de suministros, para que estas personas ejerzan el derecho a decidir libre y responsablemente, si quieren o no tener hijos, así como su número y el espaciamiento entre ellos"’3.

Teniendo en cuenta lo anterior y que es el profesional de enfermería, quien en mayor medida entra en contacto con el usuario de los programas en salud sexual y reproductiva y en quien descansa la mayor responsabilidad de la adherencia a los mismos; el objetivo del presente estudio fue identificar el cumplimiento por parte del profesional de enfermería de los aspectos técnico-científicos del programa de planificación familiar, establecidos en las normas y políticas públicas vigentes en salud sexual y reproductiva y calidad de la atención, en una Institución prestadora de servicios de salud. 


\section{Materiales y métodos}

Se efectuó un estudio descriptivo, de corte transversal; la población de estudio fueron usuarios y enfermeros del programa de planificación familiar de una institución prestadora de servicios de salud (IPS) de primer nivel de atención de la ciudad de Bucaramanga, entre octubre de 2013 a junio de 2014. La muestra estuvo constituida por 345 usuarias y 11 profesionales de enfermería, seleccionadas aleatoriamente.

Los criterios de inclusión que se tuvieron en cuenta fueron: usuarias del programa de planificación familiar atendidas en la IPS y profesionales de enfermería encargados de la atención de este programa en la IPS. Los criterios de exclusión fueron: usuarios de este programa con alteraciones mentales diagnosticadas por un especialista y que afecten la comunicación y el personal auxiliar de enfermería.

Para la recolección de la información se aplicó un instrumento diseñado por el grupo investigador teniendo en cuenta los lineamientos de obligatorio cumplimiento establecidos en la normatividad Colombiana, que permitió mediante observación verificar el cumplimiento de estándares de calidad del cuidado brindado por el profesional de enfermería en relación con la humanización del cuidado y satisfacción con la atención; cumplimiento de aspectos técnicocientíficos y disponibilidad de recursos e insumos. Los procedimientos de recolección y procesamiento de datos se realizaron en EpiData 3.1, los cuales fueron exportados al programa Stata v 12.

Esta es una investigación sin riesgo, según la resolución 008430 de 1993 del Ministerio de salud Colombiano para investigaciones con seres humanos, porque no se realizó ninguna intervención o modificación intencionada de las variables biológicas, fisiológicas o sociales de los individuos que participaron en el estudio $^{6}$. La información obtenida fue manejada bajo los principios éticos de: beneficencia, no maleficencia, autonomía y calidad, haciendo énfasis en la confidencialidad y privacidad de la información ${ }^{7}$. Se tuvo en cuenta: la aprobación por parte de los comités de ética de las instituciones involucradas en el estudio, y el consentimiento informado ${ }^{8}$ de los sujetos de investigación por escrito. Los anteriores aspectos éticos fueron respaldados por el documento del Consejo de Organizaciones Internacionales de las ciencias médicas CIOMS sobre las pautas éticas Internacionales para la investigación Biomédica en Seres Humanos?.

\section{Resultados}

De acuerdo a las características sociodemográficas de la población de estudio se encontró que el $100 \%$ eran mujeres, entre los 14 y 50 años, con una mediana de 23 años. El estado civil predominante fue unión libre 63.1\%. La mayoría de ellas pertenecían a estratos socioeconómicos bajos $1(56,6 \%)$ y $2(33,2 \%)$ respectivamente, residentes en el área urbana 93,1\%, con un nivel de escolaridad de secundaria: completa $39,1 \%$ e incompleta $31,6 \%$. Con relación al tipo de seguridad social el $100 \%$ estaba afiliada a una Entidad Promotora de Salud del régimen subsidiado, que le garantizaba la prestación de los servicios en salud.

Como se indica en la Tabla 1, sobre la Organización e infraestructura del programa de planificación familiar, el $80,9 \%$ de los consultorios en que se brinda el programa de planificación familiar no contaba con identificación exterior que facilite su ubicación y faltan mecanismos de información relacionados con el programa que se ubiquen en la parte externa del consultorio (77,3\%), así como del horario del programa en el $76 \%$ de las unidades de atención observadas. El $100 \%$ de los consultorios contaban con un ambiente de trabajo ventilado, cómodo, iluminado y limpio; privado en el $74 \%$ de los casos, aunque el $87,5 \%$ no tenían baño en el consultorio para uso de los usuarios; la mayoría contaba con recipientes para la clasificación de desechos y el $98.8 \%$ del personal de enfermería clasificaba adecuadamente los residuos.

Se evidenció una excelente disponibilidad de anticonceptivos orales $100 \%$, inyectables $(97.9 \%)$, dispositivo intrauterino DIU (77,4\%); sin embargo en el $53.6 \%$ de los casos no se contaba con preservativos. Tenían papelería como historias clínicas y formatos de entrega de anticonceptivos (96,8\%), así como consentimientos informados en un $95 \%$. Se disponía de elementos para valoración como tensiómetro $(90 \%)$, báscula $(99,7 \%)$, fonendoscopio $(96,5 \%)$, fichero para identificación de usuarios (84\%). Aunque el 87,8\% de los consultorios del programa contaban con material educativo para brindar asesoría a los usuarios, es de resaltar que un $12 \%$ no lo tenían.

Aspectos relacionados con el diligenciamiento de la información de la consulta del programa de planificación familiar, como se observa en la Tabla 2, se encontró que en el $97,9 \%(n=338)$ de las consultas los profesionales diligencian los formatos de historia clínica, según el tipo de consulta si es de primera vez o de control. 
Tabla 1. Organización e infraestructura del programa de planificación familiar.

\begin{tabular}{|c|c|c|c|}
\hline \multirow{2}{*}{ Indicadores } & \multicolumn{3}{|c|}{ Presente } \\
\hline & Si n(\%) & No $n(\%)$ & $\mathrm{NA}^{*} \mathbf{n}(\%)$ \\
\hline Identificación exterior del consultorio & $66(19,1)$ & $279(80,9)$ & --- \\
\hline \multicolumn{4}{|l|}{ Ambiente de trabajo: } \\
\hline Iluminado & $345(100)$ & ---- & ---- \\
\hline Ventilado & $345(100)$ & ---- & ---- \\
\hline Limpio & $345(100)$ & ---- & ---- \\
\hline Privado & $256(74,2)$ & $89(25,8)$ & ---- \\
\hline Existencia de sanitario para uso de la persona que consulta & $43(12,5)$ & $302(87,5)$ & ---- \\
\hline Clasificación adecuada de los desechos & $341(98,8)$ & $4(1,2)$ & ---- \\
\hline Presencia de un sistema para identificación de usuarios & $290(84,1)$ & $55(15,9)$ & ---- \\
\hline \multicolumn{4}{|l|}{ Disponibilidad de elementos para valoración de usuarios } \\
\hline Tensiómetro & $311(90,1)$ & $34(9,8)$ & ---- \\
\hline Fonendoscopio & $333(96,5)$ & $12(3,4)$ & ---- \\
\hline Peso & $344(99,7)$ & $1(0,2)$ & ---- \\
\hline Material educativo para asesoría en el programa & $303(87,8)$ & $42(12,1)$ & ---- \\
\hline Información acerca del horario del programa & $81(23,5)$ & $263(76,2)$ & $1(0,2)$ \\
\hline \multicolumn{4}{|l|}{ Disponibilidad de insumos } \\
\hline Preservativos & $160(46,4)$ & $185(53,6)$ & ---- \\
\hline Anticoncepción de emergencia & $344(99,7)$ & $1(0,3)$ & ---- \\
\hline Anticonceptivos orales & $345(100)$ & ---- & ---- \\
\hline Anticonceptivos inyectables & $338(97,9)$ & $7(2,0)$ & ---- \\
\hline DIU & $267(77,4)$ & $78(22,6)$ & ---- \\
\hline
\end{tabular}

Fuente: Elaboración propia con base en los datos analizados. *NA: No Aplica

Tabla 2. Consulta de enfermería en el programa de planificación familiar.

\begin{tabular}{|c|c|c|c|}
\hline \multirow{2}{*}{ Indicadores } & \multicolumn{3}{|c|}{ Presente } \\
\hline & Si n(\%) & No $n(\%)$ & $\mathbf{N A} \mathbf{n}(\%)$ \\
\hline \multicolumn{4}{|l|}{ a. Diligenciamiento de la información } \\
\hline Diligenciamiento de Historia clínica según tipo de consulta & $338(97,9)$ & $5(1,4)$ & $2(0,5)$ \\
\hline Consentimiento informado para inserción del implante & $340(98,5)$ & $5(1,5)$ & ---- \\
\hline Registro de hallazgos encontrados & $206(59,7)$ & $17(4,9)$ & $122(35,4)$ \\
\hline Caligrafía legible & $345(100)$ & ---- & ---- \\
\hline Firma del responsable de la consulta & $345(100)$ & ---- & ---- \\
\hline \multicolumn{4}{|l|}{ b. Desarrollo de la consulta } \\
\hline \multicolumn{4}{|l|}{ Aplicación de medidas de bioseguridad } \\
\hline Uso de bata & $65(18,8)$ & $278(80,5)$ & $2(0,5)$ \\
\hline Lavado de manos según protocolo institucional & $52(15,1)$ & $139(40,3)$ & $154(44,6)$ \\
\hline Guantes limpios o estériles según sea el caso & $2(0,5)$ & $5(1,4)$ & $338(97,9)$ \\
\hline Cabello recogido & $284(82,3)$ & $21(6,1)$ & $40(11,6)$ \\
\hline Limpieza: zona de aplicación anticonceptivo inyectable & $108(31,3)$ & $5(1,4)$ & $232(67,3)$ \\
\hline \multicolumn{4}{|l|}{ Asesoría en derechos sexuales y reproductivos: } \\
\hline Libertad sexual & $6(1,7)$ & $339(98,3)$ & ---- \\
\hline Autonomía, integridad y seguridad sexual del cuerpo & $8(2,3)$ & $337(97,6)$ & ---- \\
\hline Privacidad sexual & $1(0,3)$ & $344(99,7)$ & ---- \\
\hline Equidad sexual & $1(0,3)$ & $344(99,7)$ & ---- \\
\hline Placer sexual & ---- & $345(100)$ & ---- \\
\hline Expresión sexual emocional & ---- & $345(100)$ & ---- \\
\hline Libre asociación sexual & $1(0,3)$ & $344(99,7)$ & ---- \\
\hline Toma de decisiones reproductivas, libres y responsables & $25(7,2)$ & $320(92,7)$ & ---- \\
\hline Información basada en el conocimiento científico & $186(54)$ & $159(46,0)$ & ---- \\
\hline Educación sexual integral & $88(25,5)$ & $257(74,5)$ & ---- \\
\hline Atención de la salud sexual & $93(27)$ & $252(73)$ & ---- \\
\hline Informar sobre doble protección, ITS, VIH/SIDA & $54(15,6)$ & $290(84,1)$ & $1(0,3)$ \\
\hline Importancia de la citología cervico-uterina. & $136(39,4)$ & $209(60,6)$ & ---- \\
\hline Importancia del auto examen de mama & $34(9,9)$ & $311(90,1)$ & ---- \\
\hline Demanda inducida para la citología cervico-uterina & $160(46,4)$ & $148(43)$ & $37(10,7)$ \\
\hline
\end{tabular}

Fuente: Elaboración propia con base en los datos analizados *NA: No Aplica 
Con relación al diligenciamiento en la historia clínica de los hallazgos, complicaciones u observaciones sobre el estado de salud de las usuarias, en el 35\% de los casos $(\mathrm{n}=122)$ no se aplicó este indicador, porque en las usuarias no se presentó este tipo de hallazgos o alteraciones. Del total de las usuarias que si presentaron hallazgos y por lo tanto aplicaba el indicador $(\mathrm{n}=$ 223 ), se identificó que el $92.3 \%$ de los profesionales de enfermería describieron en las historias clínicas los respectivos hallazgos, sin embargo es de destacar que el $7.6 \%$ de los casos en que sí debían registrar los hallazgos encontrados, no lo hicieron.

Un componente importante en el cuidado de enfermería que se brinda en un programa de planificación familiar, es el desarrollo de la consulta, del cual se encontró en este estudio, que en el $44,6 \%$ de las consultas( $n=154$ ), no se aplicó el indicador de lavado de manos según el protocolo institucional, porque eran consultas relacionadas con anticonceptivos hormonales orales, que no requerían del procedimiento. No se realizó el lavado de manos en el 72,7\%(n=139) de los casos que si ameritaba, es decir $n=191$ en total; solo se hizo el lavado de manos de forma adecuada en el 27,2\%(n=52).

En el $81 \%$ de los casos, los enfermeros no utilizaron la bata para realizar la consulta de planificación familiar. Dado que los anticonceptivos más utilizados fueron orales e inyectables no se requirió el uso de guantes en el $97,9 \%$ de las consultas. En el 4,42\% de la consultas que si requerían ( $\mathrm{n}=113)$, no se realizó limpieza de la zona de aplicación del anticonceptivo hormonal inyectable.

No se brindó asesoría en derechos sexuales y reproductivos en el $98 \%$ de los casos. Al $84 \%$ de las usuarias no se les informó sobre el uso del preservativo como método de doble protección, para la anticoncepción y la prevención de infecciones de transmisión sexual (ITS). Asimismo faltó que les informaran sobre la importancia de realizarse periódicamente la citología cervico vaginal $(60,6 \%)$ y del autoexamen de mama (90\%).

Los hallazgos de la valoración de los usuarios en la consulta de control del programa de planificación familiar, se muestran en la Tabla 3, se indagó por la presencia de signos y síntomas significativos asociados al uso de métodos anticonceptivos en los 339 casos que lo ameritaban como: amenorrea $(89,6 \%)$, cefalea $(85,2 \%)$, entre otros; sin embargo en un porcentaje representativo de casos no se valoró la presencia de signos relevantes como: edema (38\%), acné (39\%), várices (35,3\%). En el $77 \%$ de las consultas los enfermeros no calcularon el Índice de Masa Corporal de las usuarias y al 34,5\% no les tomaron la presión arterial.

Tabla 3. Elementos de la valoración a usuarios de planificación familiar.

\begin{tabular}{|c|c|c|c|}
\hline \multirow{2}{*}{ Indicadores } & \multicolumn{3}{|c|}{ Presente } \\
\hline & Si n(\%) & No n(\%) & $\mathrm{NA}^{*} \mathbf{n}(\%)$ \\
\hline \multicolumn{4}{|c|}{ En la consulta de control del método anticonceptivo se tiene en cuenta: } \\
\hline Tipo de método & $344(99,7)$ & $1(0,3)$ & ---- \\
\hline Edad & $344(99,7)$ & $1(0,3)$ & ---- \\
\hline Tensión Arterial & $226(65,5)$ & $119(34,5)$ & ---- \\
\hline Peso & $340(98,5)$ & $5(1,5)$ & ---- \\
\hline Índice de masa corporal & $78(22,6)$ & $267(77,4)$ & ---- \\
\hline Cefalea & $289(84)$ & $50(14,5)$ & $6(1,7)$ \\
\hline Mareo & $278(80,6)$ & $61(17,7)$ & $6(1,7)$ \\
\hline Manchas en piel & $225(65,2)$ & $114(33)$ & $6(1,7)$ \\
\hline Náuseas & $263(76,2)$ & $76(22)$ & $6(1,7)$ \\
\hline Dismenorrea & $285(82,6)$ & $54(15,6)$ & $6(1,7)$ \\
\hline Amenorrea & $304(88,1)$ & $35(10,1)$ & $6(1,7)$ \\
\hline Edema & $209(61)$ & $130(37,7)$ & $6(1,7)$ \\
\hline Várices & $219(63,5)$ & $120(34,8)$ & $6(1,7)$ \\
\hline Mastalgia & $226(65,5)$ & $113(32,7)$ & $6(1,7)$ \\
\hline Galactorrea & $224(64,9)$ & $115(33,3)$ & $6(1,7)$ \\
\hline Acné & $207(60)$ & $132(38,3)$ & $6(1,7)$ \\
\hline Leucorrea & $235(68,1)$ & $104(30,1)$ & $6(1,7)$ \\
\hline Efectos adversos & $235(68,1)$ & $104(30,1)$ & $6(1,7)$ \\
\hline Criterios de elegibilidad del método anticonceptivo & $22(6,4)$ & $4(1,1)$ & $319(93)$ \\
\hline
\end{tabular}

Fuente: Elaboración propia con base en los datos analizados *NA: No Aplica 
En 26 casos en que era necesaria la aplicación de los criterios de elegibilidad, se dio cumplimiento a este ítem en un 84,6\% (6 consultas); sin embargo es de resaltar que en el 15,38\% (cuatro casos) no los aplicaron; siendo que estos criterios de elegibilidad se deben aplicar en el $100 \%$ de los casos que lo requieran.

\section{Discusión}

De las características de la infraestructura y organización, identificadas en los consultorios del programa de planificación familiar, se encontró que la mayoría de éstos, les faltaba información exterior sobre este programa $(80,9 \%)$, siendo un aspecto importante en la prestación del servicio, pues con base en lo planteado por Muñoz, et al. ${ }^{10}$ Una de las funciones esenciales de la salud pública en los países de las Américas consiste en evaluar y promocionar el acceso equitativo de la población a los servicios de salud necesarios, por lo que los profesionales de la salud deben facilitar la vinculación de grupos vulnerables a los servicios de salud y a los servicios de educación para la salud, promoción de la salud y prevención de enfermedades. De tal manera, que si no se tiene identificado el consultorio de planificación familiar y tampoco existen mecanismos que brinden información a las personas, imposibilita que estas acudan a solicitar una consulta o que desconozcan la existencia de dicho programa.

Teniendo en cuenta que la Resolución 2003 del Ministerio de Salud Colombiano de $2014^{11}$, relacionada con estándares y criterios de habilitación sobre la infraestructura, precisa que las condiciones de orden, aseo, limpieza y desinfección deben ser evidentes y responder a un proceso dinámico de acuerdo a los servicios prestados por la institución; en este estudio se identificó, que los consultorios tienen un ambiente de trabajo tranquilo, iluminado y ventilado, lo cual coincide con lo reportado por un estudio similar realizado en Nicaragua ${ }^{12}$ en el cual el 100\%, cumplía con estas condiciones. También se identificó un ambiente ventilado, cómodo, ordenado, limpio y privado. Sin embargo, no disponen de un sanitario exclusivo para los usuarios del programa en el 87,5\% de los casos, aspecto que es fundamental teniendo en cuenta el ámbito de intimidad que implica la atención en salud sexual y reproductiva.

Los consultorios cuentan con los estándares de habilitación mínimos para la prestación de servicios de salud enmarcados en la normatividad vigente ${ }^{11}$, puesto que se observó que cuentan con los elementos requeridos para registrar información relacionada con la atención, con la valoración y con la disponibilidad de material educativo, lo cual coincide con otro estudio realizado en Brasil ${ }^{13}$, en el cual el $62.7 \%$ cuentan con material educativo, destacando, que la disponibilidad de este material es esencial para contribuirle al usuario en la elección del método. Por lo tanto, la falta de materiales educativos de buena calidad puede influir negativamente en la comprensión y elección por parte de los usuarios del método de planificación familiar.

Al indagar acerca de si la institución cuenta con insumos como preservativos, se encontró que en el $53,6 \%$ de las consultas no se disponía de dicho método de barrera, siendo su uso esencial para la prevención de enfermedades de transmisión sexual y gravidez ${ }^{14}$. Según la Organización Mundial de la Salud OMS ${ }^{15}$, en muchos países de bajos y medianos ingresos escasean o no existen métodos anticonceptivos, como preservativos, debido a que las leyes y las políticas son inadecuadas, los sistemas de suministro y la gestión de la logística o la financiación es insuficiente o no existe.

Lo anterior se relaciona con los datos del estudio de Valencia, et al. ${ }^{16}$ quienes identificaron que los jóvenes adquieren el condón en mayor proporción en las farmacias y menos en los servicios de salud. Esto evidencia las falencias en el cumplimiento de algunas disposiciones legales en el marco de los servicios de salud como la distribución de condones a jóvenes con vida sexual activa. Tal como lo declaran ONUSIDA y UNFPA $^{17}$, los preservativos deben estar fácilmente disponibles universalmente, con carácter gratuito o a un precio razonable y fomentarse de un modo tal que ayude a salvar los obstáculos sociales y personales de su uso.

En los resultados del estudio, el profesional de enfermería en la mayoría de los casos no informó sobre la importancia de usar doble protección, como método de anticoncepción y prevención de ITS, incluida el VIH/SIDA. Es necesario romper con la creencias asociadas a falta de conocimiento sobre el uso del condón, tal como lo afirma Soto $\mathrm{V} \cdot{ }^{18}$ quien identificó en su estudio, que no utilizaban el condón principalmente porque: los hombres referían falta de disponibilidad del mismo en el momento de la relación sexual, que disminuía el placer y las mujeres consideraban que se perdía el romanticismo, y no se encontraba utilidad o ventaja; debido a que preferían otro método de planificación familiar. Por lo tanto la información que tiene el usuario, sobre el método anticonceptivo que va a utilizar con su pareja influye en la continuidad de su uso ${ }^{19}$. 
Teniendo en cuenta lo anterior, es fundamental que el profesional de enfermería realice la gestión para la disponibilidad de preservativos y brinde educación a las personas que acuden a los programas de control de la fecundidad, se debe abordar el riesgo de ITS y la necesidad de usar el preservativo como método de doble protección sexual.

En este estudio, se evidenció que en los consultorios se realiza una adecuada gestión de residuos, consistente en la clasificación y disposición en los recipientes y contenedores adecuados, de acuerdo a la resolución sobre gestión integral de residuos hospitalarios y similares ${ }^{20}$.

Durante el desarrollo de las consultas, se identificó que la mayoría de los enfermeros, cumple con la normatividad establecida para el diligenciamiento de la historia clínica, es decir realiza los registros sin tachones teniendo en cuenta nombres y apellidos, número de historia clínica, tipo de seguridad social, edad del usuario, acorde con las exigencias legales vigentes $^{21}$ para el manejo de la historia clínica y los registros. Uno de los elementos que esta norma exige es el consentimiento informado, el cual fue solicitado por la mayoría del personal de enfermería, previo a la realización de las intervenciones de cuidado, con el objeto de que conozcan su conveniencia y posibles efectos no deseados, a fin de que puedan manifestar su aceptación o su oposición a ellas, para los métodos anticonceptivos que así lo requirieron (ejemplo: implante subdérmico y métodos definitivos).

El diligenciamiento adecuado de la historia clínica, es un aspecto esencial dentro de la calidad de la atención, que respalda al profesional de enfermería ante cualquier situación de tipo jurídico-legal, aspecto que se cumple en el presente estudio y que en algunos casos no se aplica como se demostró en una investigación desarrollada en Cartagena ${ }^{22}$, donde el $23 \%$ de los enfermeros no registran la evolución del paciente durante el turno.

En cuanto a la aplicación de las medidas de bioseguridad, el lavado de manos es la forma más eficaz de prevenir la infección cruzada entre paciente, personal hospitalario y visitantes ${ }^{23}$, sin embargo no se llevó a cabo por los enfermeros del estudio, en muchos de los casos $72,7 \%(\mathrm{n}=139)$ que requerían este procedimiento, que debe realizarse en condiciones específicas y según lo determinado por los protocolos institucionales, con el fin de proteger la salud del enfermero y la persona de cuidado, ya que la higiene de las manos está considerada dentro de las precauciones universales de bioseguridad, según lo recomendado por la $\mathrm{OMS}^{24}$.
De acuerdo a los resultados del estudio, en el $80,58 \%$ de los casos no se utilizó la bata, lo cual se asimila al estudio realizado por Rojas, et al. ${ }^{25}$ en Mérida Venezuela acerca de conocimiento y aplicación sobre medidas de bioseguridad en el personal médico y de enfermería, en el cual se identificó que el $87 \%$ de este personal no utilizó: bata, guantes, ni tapabocas entre otras.

Es de recordar que los Elementos de Protección Personal (EPP), crean una barrera que mantiene a la persona fuera del contacto con los peligros (físicos, químicos, biológicos, mecánicos) que puedan estar presentes en un ambiente de trabajo, la bata es uno de ellos y por tanto es obligación del trabajador el uso y mantenimiento adecuado de los mismos según lo dispuesto por la normatividad nacional ${ }^{26}$.

Durante la mayoría de las consultas del estudio, no se brindó asesoría en derechos sexuales y reproductivos, siendo éstos una estrategia fundamental planteada por la ONU en la Conferencia Internacional sobre Población y Desarrollo (El Cairo 1994) para la reducción de la pobreza, la mejoría de la salud y la calidad de vida de las personas ${ }^{27}$, propuesta que sigue siendo vigente más de veinte años después; es así como los esfuerzos de los diferentes países del mundo en el marco de los Objetivos de desarrollo sostenible (ODS 2030), reconocen que los cambios demográficos son el resultado de las elecciones individuales, y que se abordan mejor a través de políticas que amplíen estas opciones y oportunidades, centrándose en la información y los servicios de salud reproductiva, que facultan a las mujeres para que participen plenamente en la vida social, económica y política ${ }^{28}$. De esta manera se torna imprescindible para Colombia la aplicación y fortalecimiento de los diferentes lineamientos en materia de salud sexual y reproductiva descritos desde la política nacional de Salud Sexual y Reproductiva (SSR) de los adolescentes, promulgada por el Ministerio de Protección Social de Colombia en el año 2003, la estrategia de servicios amigables para jóvenes y adolescentes y el Plan Decenal de Salud Pública, entre otros ${ }^{29}$, en los cuales se promueve el mejoramiento de la SSR, la promoción y el ejercicio de los Derechos Sexuales y Reproductivos DSR de toda la población.

Situación similar se encontró en el estudio realizado por la Alcaldía de Medellín, acerca del impacto del programa de salud sexual y reproductiva en adolescentes ${ }^{30}$, donde se encontró que una de las causas institucionales del embarazo adolescente en esta población es el déficit de los servicios específicos de consejería sobre salud sexual y reproductiva además de la falta de promoción de los derechos sexuales y reproductivos. 
Es por ello, que el personal de enfermería debe hacer uso de las estrategias y espacios existentes en el ejercicio de su profesión, para garantizar a los sujetos de cuidado el acceso a la información con un enfoque de género basado en los DSR buscando ser garantes de los mismos. En concordancia, deben establecerse estrategias que promuevan acciones de información, educación y comunicación ${ }^{31}$, donde participen no sólo los jóvenes sino también las redes sociales e instituciones de las que ellos hacen parte como son servicios de salud, sus pares, los padres de familia, los docentes y las organizaciones juveniles.

Ligado a la información de un método anticonceptivo y de los derechos sexuales y reproductivos, debe ir la asesoría sobre la importancia de la realización periódica del autoexamen de mama y de la citología cervicouterina, la cual no se tuvo en cuenta en el $90 \%$ y $60,5 \%$ de los casos del estudio respectivamente, como parte integral del autocuidado de la mujer, ya que el autoexamen de mama es un pilar fundamental en el diagnóstico precoz del cáncer de mama ${ }^{32-33}$, pues es el método de pesquisa eficaz, inocuo, que está al alcance de todas las mujeres y no requiere de recursos económicos para su realización.

Asimismo la citología es un examen de bajo costo, sencillo, con gran especificidad en el diagnóstico de las displasias y diversos microorganismos patógenos vaginales, lo que la convierte en una gran prueba de tamizaje $\mathrm{e}^{34-35}$, es decir de gran utilidad en la detección de infecciones y reducción de muertes por cáncer a nivel cervicouterino. Se ha identificado que los cambios hormonales que la mujer tiene como producto del uso de los anovulatorios la tornan más susceptible a neoplasia cervical, según estudio realizado en el $\mathrm{Cauca}^{36}$, en que el uso de anticonceptivos hormonales fue mayor en las mujeres con resultado de citología anormal (61\%) en comparación con citología normal (47\%) y el uso de anticonceptivos hormonales durante más de 5 años aumenta el riesgo hasta cuatro veces de desarrollar un carcinoma invasor de cérvix. Un estudio realizado en $\mathrm{Chile}^{37}$, concluyó que los ACO provocarían un aumento en la incidencia de ectropión cervico uterino, favoreciendo una mayor exposición de la unión escamocolumnar a potenciales agentes carcinogénicos.

Igualmente De Villiers ${ }^{38}$, ha reportado que el estrógeno y la progesterona también podrían afectar directamente las células cervicouterinas, aumentando la proliferación celular y estimulando la actividad transcripcional (transactivación) de los oncogenes E6 y E7 del virus del papiloma humano VPH. Por consiguiente, a las mujeres que utilizan anticonceptivos hormonales se les debe realizar un seguimiento más riguroso en las consultas de planificación familiar, así como el control de los resultados de la citología.

Teniendo en cuenta que el desconocimiento y el temor según Del Toro, et al. ${ }^{39}$, son las principales razones por las cuales las mujeres no se realizan el autoexamen o lo hacen de manera incorrecta; así mismo, el desconocimiento y temor también aplica para la realización de la citología. Es por ello que le corresponde al profesional de enfermería asumir un compromiso decidido para la resolución de dichos aspectos que inhiben estos mecanismos de prevención secundaria y de esta manera favorecer la adherencia a estos programas de detección temprana de neoplasias en la mujer.

Es de vital importancia resaltar la necesidad que los enfermeros realicen una valoración detallada por medio del examen físico de los usuarios del programa de planificación familiar, para determinar posibles efectos secundarios de los métodos anticonceptivos, en razón a que se evidenció en el presente estudio que entre el 30$39 \%$ de los casos, no se valoró la presencia de signos importantes como: edema, manchas en la piel, várices, acné, así como en la mayoría, no se calculó el Índice de Masa Corporal, utilizado para evaluar el estado nutricional, aunque si realizaron la medición del peso, del cual está demostrado que la ganancia del mismo, es uno de los efectos secundarios frecuentes de los anticonceptivos hormonales ${ }^{40}$. Al 34.5\% no les tomaron la presión arterial, siendo este signo fundamental sobre todo en las mujeres con antecedentes familiares de hipertensión arterial, que pueden desarrollar hipertensión secundaria al uso de anticonceptivos orales; cuando son hipertensas es importante la evaluación de la causa y el nivel de la hipertensión.

Igualmente, es esencial informar a las mujeres que inician la anticoncepción hormonal de las posibles reacciones adversas que pudieran aparecer y ayudar en lo posible a su prevención, teniendo en cuenta los lineamientos de la política Colombiana en materia de seguridad del paciente ${ }^{41}$.

También se deben tener en cuenta los criterios de elegibilidad del método anticonceptivo de la $\mathrm{OMS}^{42}$, porque aunque en el estudio la mayoría de consultas fueron de control y no cambiaron de método, de los 26 casos que si ameritaban la aplicación de estos criterios, en cuatro casos $(15,38 \%)$ no los tuvieron en cuenta, siendo vital la aplicación de estos criterios en el 100\% de los casos que lo ameriten; ya que desde el punto 
de vista de las mujeres, las elecciones de los métodos anticonceptivos se realizan dentro de un marco temporal, social y cultural. Las elecciones son complejas, multifactoriales y están sujetas a cambios, por lo cual se debe tener en cuenta condiciones importantes de la mujer como: historia reproductiva, enfermedad cardiovascular, desórdenes e infecciones del tracto reproductivo, condiciones neurológicas, endocrinas, gastrointestinales e interacciones farmacológicas; además de las diferentes características del método, ya que la toma de decisiones del mismo está sujeta a las circunstancias e interpretaciones individuales.

En conclusión de acuerdo a los resultados obtenidos, se identificó que las debilidades técnico científicas, que se presentaron en la atención del programa de planificación familiar por parte de enfermería, estaban relacionadas con la falta de información a las usuarias sobre sus cuidados sexuales y reproductivos, los derechos relacionados con estos cuidados y la valoración completa del examen físico, para identificar cualquier alteración del estado de salud.

De igual forma se destaca el compromiso del personal de enfermería, en dos aspectos importantes el cumplimiento con el diligenciamiento completo de las historias clínicas del programa de planificación familiar y la aplicación de los aspectos éticos en la atención a los usuarios, evidenciados por la utilización de los consentimientos informados para la inserción, retiro de los dispositivos intrauterinos e implantes subdérmicos y en la satisfacción del usuario con la atención.

Es necesaria la adopción de un plan de mejoramiento acorde con los hallazgos del presente estudio, que favorezca la calidad del programa de planificación familiar, teniendo en cuenta el liderazgo que el profesional de enfermería posee en el desarrollo $\mathrm{y}$ adherencia de los usuarios a este mecanismo de prevención primaria empleado en el mundo y de obligatorio cumplimiento en Colombia, que permite la vivencia de una sexualidad en forma responsable, sana y gratificante.

Es fundamental que el profesional de enfermería continúe la realización de estudios sobre la evaluación de aspectos técnico científicos, relacionados con la calidad de la atención en salud, lo cual contribuye a enriquecer su conocimiento y quehacer, sin desconocer la importancia de la humanización del cuidado y la satisfacción del usuario, que también son propios del interés y centro de atención de enfermería.

\section{Agradecimientos}

A la Vicerrectoría de Investigación y Extensión, de la Universidad Industrial de Santander, por el apoyo financiero para el desarrollo de este trabajo.

\section{Consideraciones éticas}

Se tuvo en cuenta la aprobación por parte de los comités de ética de las instituciones involucradas en el estudio y el consentimiento informado de los sujetos de investigación por escrito.

\section{Conflicto de interés}

Las autoras expresamos que no hemos incurrido en conflicto de interés alguno que ponga en riesgo la integridad de la información aquí presentada y que durante la ejecución del trabajo, así como durante la redacción del artículo, no han incidido intereses o valores distintos a los que usualmente tiene la investigación.

\section{Referencias}

1. United Nations Population Information Network (POPIN). UN Population Division, Department of Economic and Social Affairs, with support from the UN Population Fund (UNFPA). Conferencia Internacional sobre la Población y el Desarrollo, El Cairo (Egipto) 1994.

2. Ministerio de la Protección Social. Sistema Obligatorio de Garantía de Calidad de la Atención de Salud del Sistema General de Seguridad Social en Salud. Decreto 1011; Colombia. 2006.

3. Ministerio de la protección social. Norma técnica para la atención en planificación familiar a hombres y mujeres: Resolución 0412 Colombia. 2000.

4. Congreso de Colombia. Reforma del Sistema general de seguridad social en salud y se dictan otras disposiciones. Ley 1438; 2011.

5. Ministerio de salud y Protección Social. Registro de las actividades de Protección Específica, Detección Temprana y la aplicación de las Guías de Atención Integral para las enfermedades de interés en salud pública de obligatorio cumplimiento Resolución 4505; Colombia. 2012.

6. Ministerio de Salud Normas científicas, técnicas y administrativas para la investigación en salud, Resolución 8430, Colombia 1993.

7. Manzini J. Declaración de Helsinki, principios éticos para la investigación médica sobre sujetos humanos, 
programa de bioética. Universidad Nacional Mar de alta. Argentina. 2000.

8. Tribunal Nacional Ético de Enfermería. Responsabilidad deontológica para el ejercicio de la profesión de enfermería en Colombia, se establece el régimen disciplinario correspondiente. Ley 911. Colombia. 2004.

9. Consejo de Organizaciones Internacionales de las Ciencias Médicas (CIOMS), Pautas éticas Internacionales para la investigación biomédica en seres humanos, Ginebra. 2002: p. 12,64.

10. Muñoz F, López D, Halverson P, Guerra C, Hanna $\mathrm{W}$, Larrieu $\mathrm{M}$ et al. Las funciones esenciales de la salud pública: un tema emergente en las reformas del sector salud. Pan Am J Public Health. 2000; 8 (1): 126-134.

11. Ministerio de Salud y Protección Social. Procedimientos y condiciones de inscripción de los Prestadores de Servicios de Salud y de habilitación de servicios de salud. Resolución 2003. Colombia. 2014.

12. Urbina D M V, Pérez H JO, Gómez A T. Calidad de Atención que brinda el personal de enfermería, desde Opinión de las usuarias del programa de planificación familiar en el Centro de Salud Carlos Lacayo Manzanares, 11 semestre 2015. Universidad Nacional Autónoma de Nicaragua; 2016.

13. Santana de Sá Fiuza É, Fagundes Durães Rocha J, Almeida Carneiro J, Marques da Costa F, Planejamento familiar: avaliação da qualidade nas dimensões da estrutura, organização e assistência. Rev Pesquisa Cuid Fund Online. 2015: 7(4): 32273238

14. Falcão J, Rabelo S, Lopes E, Freitas L, Pinheiro A, Ximenes L. Perfil e práticas sexuais de universitários da área da saúde. Esc Anna Nery R Enferm. 2007; 11(1): 58-65.

15. Organización Mundial de la Salud. Respeto de los derechos humanos al proporcionar información y servicios de anticoncepción: orientaciones y recomendaciones. 2014.

16. Valencia C, Canaval G. Factores que predisponen, facilitan y refuerzan el uso del preservativo en jóvenes universitarios de Cali, Colombia. Rev Sal Pública. 2012; 14(5): 810-821.

17. ONUSIDA, UNFPA. Los preservativos y la prevención del VIH: declaración de ONUSIDA, UNFPA y OMS. 2009

18. Soto V. Factores asociados al no uso del condón: Estudio en adolescentes y adultos jóvenes en Chiclayo. An Fac Med. 2006; 67 (2): 152-159. DOI: http://dx.doi.org/10.15381/anales.v67i2.1253

19. Labrada M, Reyes W, Megret O, Dieppa O.
Influencia de Aspectos Bioéticos en La Planificación Familiar en Santos Suárez. Rev Cubana Med Gen Integr 1999;15(4): 378-385.

20. Mora C, Berbeo M. Instituto Nacional de Salud. Subdirección Red Nacional de Laboratorios SRNL. Manual de Gestión Integral de Residuos. 2010. p. 5-57.

21. Ministerio de Salud. Normas para el manejo de la historia clínica. Resolución 1995. Colombia.1999.

22. Fernández S, Díaz A, López $\mathrm{C}$, Villa Y. Evaluación de la calidad de las notas de enfermería en el área de hospitalización. Rev Ciencia Sal Virtual. 2012; 4(1): 86-92. DOI: https://doi. org/10.22519/21455333.250.

23. Ministerio de Salud. Dirección General de Promoción y Prevención: Programa Nacional de Prevención y Control de las ETS/VIH/SIDA. Conductas Básicas en Bioseguridad: Manejo integral. Protocolo Básico para el equipo de salud. Bogotá; 1997.

24. Organización Mundial de la Salud. Campaña mundial Anual "Salve vidas: Límpiese las manos". Momentos para la Higiene de las manos.

25. Rojas L, Flores M, Berrios M, Briceño I, Conocimiento sobre medidas de bioseguridad en personal médico y de enfermería. Mérida. Venezuela. MedULA; 2013: 22: 33-40.

26. Código Sanitario Nacional, Ley 9, Art.85.Congreso de Colombia, reglamentado en 1979 y 2008 por decreto nacional 2493.

27. Galdos S. The Cairo conference and the assertion of sexual and reproductive rights as a basis for sexual and reproductive health. Rev Per Med Experim Sal Pública. 2013; 30(3): 455-460.

28. Herrmann M. Sustainable development, demography and sexual and reproductive health: inseparable linkages and their policy implications. Reproductive Health Matters 2014; 22(43): 28-42. DOI: 10.1016/S0968-8080(14)43757-1.

29. Ministerio de salud y protección social. Plan decenal de salud pública. 2012-2021: la salud en Colombia la construyes tú. Colombia 2011.

30. Alcaldía de Medellín, Secretaría de salud. Evaluación del impacto del programa de salud sexual y reproductiva en adolescentes de Medellín. Fase cualitativa. Medellín, Colombia. 2008. p.1-130.

31. Vásquez M, Argote L, Castillo E, Mejía M, Tunjo R, Villaquirán M, Educación en derechos sexuales $\mathrm{y}$ reproductivos: una perspectiva integral con adolescentes escolarizados. Colomb Med. 2005; 36(1): 6-13.

32. Gálvez M. Relación entre el conocimiento del autoexamen de mama y la prevención del cáncer de mama. Mediciego 2013; 19 (2). 
33. González M, González L, Caballero M, Aguilar M. Formación de médicos y enfermeras para la detección temprana del cáncer de mama en México. Rev Salud Pública. 2011: 13 (6): 966-979.

34. Rivera B, Quintero J. Prevalencia de lesiones intraepiteliales escamosas y factores de riesgo en las usuarias del servicio de citologías del laboratorio docente asistencial, proyecto de investigación, Escuela de Bacteriología, Universidad de Antioquia, Medellín 2006.

35. Fajardo M, García A., Caballero M., Vargas D., Camargo F., Prevalencia y factores asociados al resultado anormal de la citología vaginal en estudiantes universitarias, en Bucaramanga Colombia. Rev Costarr Salud Pública. 2013; 22: 127-133.

36. Tafurt Y. Acosta C., Sierra C., Prevalencia de citología anormal e inflamación y su asociación con factores de riesgo para neoplasias del cuello uterino en el Cauca, Colombia. Rev Salud pública. 2012; 14 (1): 53-66.

37. Solís MT, Aguayo F, Vargas M, Olcay F, Puschel K, Corvalán A, et al. Rev Méd Chile. Santiago de Chile. 2010; 138(2): 175-180. DOI: http://dx.doi. org/10.4067/S0034-98872010000200005.

38. De Villiers EM. Relationship between steroid hormone contraceptives and HPV, cervical intraepithelial neoplasia and cervical carcinoma. Int I Cáncer 2003; 103: 705-708. DOI: 10.1002/ ijc. 10868 .

39. Del Toro RM, Peinado VL, Díaz PA. Conocimientos y prácticas sobre el autoexamen mamario en mujeres de Cartagena de Indias (Bol.-Col). Cienc Innov Salud. 2014; 2 (1): 27-32.

40. Arrate M, Linares M, Molina V, Sánchez N, Arias $\mathrm{M}$, Efectos secundarios de los anticonceptivos hormonales en usuarias del método asistentes a las consultas de planificación familiar. MEDISAN 2013; 17(3): 415-427.

41. Ministerio de Protección Social. Lineamientos para la implementación de la política de seguridad del paciente. Colombia. 2008. p.1-49.

42. Organización Mundial de la Salud. Criterios médicos de elegibilidad para el uso de anticonceptivos, Cuarta edición, 2009: Un pilar de la OMS para la planificación familiar. Ginebra 2012, OMS. 